\title{
Label-Free Detection of Oligosaccharide-Lectin Interaction Using Plasmonic Optical Device for Glycomics Application
}

\author{
Tatsuro Endo ${ }^{1,2, *}$, Sayoko Matsuda ${ }^{3}$, Takakiyo Obara ${ }^{3}$, Yasushi Chuma ${ }^{3}$, \\ Makoto Ogata $^{3}$, Yasuko Yanagida ${ }^{1,2}$, Takeshi Hatsuzawa ${ }^{1,2}$ and Taichi Usui ${ }^{3}$ \\ 'Department of Mechano-Micro Engineering, \\ Interdisciplinary Graduate School of Science and Engineering, Tokyo Institute of Technology, \\ 4259 Nagatsuta-cho, Midori-ku, Yokohama 226-8502, Japan \\ ${ }^{2}$ Precision and Intelligence Laboratory, Tokyo Institute of Technology, \\ 4259 Nagatsuta-cho, Midori-ku, Yokohama 226-8503, Japan \\ ${ }^{3}$ Department of Applied Biological Chemistry, Faculty of Agriculture, Shizuoka University, \\ Ohya 836, Suruga ward, Shizuoka 422-8529, Japan
}

(Received March 2, 2010; accepted June 1, 2010)

Key words: plasmonics, localized surface plasmon resonance (LSPR), oligosaccharide, lectin, glycomics

In this paper, we describe the fabrication and characterization of a localized surface plasmon resonance (LSPR)-based plasmonic optical device for the label-free detection of oligosaccharide-lectin interaction using a core-shell-structured nanoparticle-layer substrate. The oligosaccharide performs a crucial function for life phenomena such as cell growth, differentiation, and canceration. To understand these functions of living organisms in terms of oligosaccharides, a more simplified, cost-effective, and labelfree analysis device has been desired. To achieve this, we aimed to develop a novel device for the detection of oligosaccharide-lectin interactions using an LSPR-based plasmonic optical device. For the detection of oligosaccharide-lectin interactions, the divalent $N$-acetyllactosamine (LacNAc) glycoside carrying a disulfide group was obtained using chemoenzymatic synthesis. Additionally, the divalent glycoside was immobilized onto the plasmonic optical device surface. Then, the changes in optical characteristics with the LacNAc glycoside-wheat germ agglutinin (WGA) interactions were determined. As a result, the LSPR-based plasmonic optical device could be used to detect oligosaccharide-lectin interactions. Furthermore, the LacNAc moiety, which was obtained using chemoenzymatic synthesis, was specifically recognized by WGA molecules. This plasmonic optical device was more simplified, requiring smaller sample volumes than those required by conventional analytical systems such as surface plasmon resonance (SPR) and a quartz crystal microbalance (QCM). Thus, the plasmonic optical

${ }^{*}$ Corresponding author: e-mail: endo.t.ab@m.titech.ac.jp 
device has potential for use in cost-effective, highly simplified, and highly sensitive test kits for future glycomics applications.

\section{Introduction}

Analyses of biomolecular interactions such as DNA-DNA hybridization, and antigen-antibody reactions are important for understanding functions of living organisms. (1) In addition, the results of such analyses have been applied to omics research such as genomics, ${ }^{(2)}$ proteomics, ${ }^{(3)}$ and cellomics. ${ }^{(4)}$ Among the abovementioned omics research, glycomics, which is focused on oligosaccharides, has been studied energetically worldwide, ${ }^{(5)}$ because oligosaccharides play a role in living organisms as signal molecules. However, for the analysis of oligosaccharide-based biomolecular interactions, there are some difficulties in the determination of kinetics such as interaction efficiency. Thus, in the conventional analysis systems and devices such as surface plasmon resonance (SPR) ${ }^{(6)}$ and the quartz crystal microbalance $(\mathrm{QCM}),{ }^{(7)}$ sophisticated techniques have been required. From these backgrounds, for glycomics applications, development of a novel analysis system and related devices has important biological implications. For the development of an analysis system and related devices that fulfill all the above-mentioned requirements, we focused on the nanostructure-based specific optical characteristics of a plasmonic optical device.

Plasmonics is an emerging research field based on using the localised surface plasmon resonances (LSPRs) of metal nanomaterials such as nanoparticles. Nanotechnology involving the use of nanomaterials such as nanotubes, nanoparticles, and nanofibers is a novel and interesting field of research. ${ }^{(8-10)}$ Using LSPR, several theoretical applications have been studied. Noble metal nanoparticles such as gold and silver are sensitive to changes in the surrounding environment, such as in the refractive indices of different media. Furthermore, they can be specifically observed in the visible region (380-750 $\mathrm{nm}$ ) of the absorption spectra owing to their property of LSPR absorption. ${ }^{(11,12)}$ Hence, using these optical properties, plasmonics is widely used in sensing applications. These features enable the establishment of a simple and cost-effective experimental setup for sensing applications. On the basis of these specific optical characteristics and experimentally determined features of plasmonics, many researchers have investigated their use in sensing applications. ${ }^{(13,14)}$

Using plasmonics-based optical characteristics, we have previously achieved the fabrication of a plasmonics optical device with a core-shell nanoparticle layer as the substrate $^{(15-20)}$ for the label-free detection of antigen-antibody reactions, cell metabolites, and peptide nucleic acid (PNA)-DNA and DNA-DNA hybridizations. In addition, the fabrication of multiple detection principle-based biosensors could be achieved. This substrate for LSPR excitation was fabricated without the use of sophisticated techniques such as those required for noble metal nanoparticle synthesis. From these achievements of plasmonic devices for biosensing applications, we aimed to detect the oligosaccharidelectin interactions for future glycomics applications.

In this study, considering the above-mentioned properties of an LSPR-based plasmonic optical device using a core-shell-structured nanoparticle-layer substrate, we 
attempted to detect the oligosaccharide-lectin interactions. We fabricated an LSPRbased plasmonic optical device and characterized it using a UV-VIS spectrophotometer. Furthermore, for specific detection of the oligosaccharide-lectin interactions, divalent disaccharide glycosides were synthesized using the chemoenzymatic synthesis method. Furthermore, using spacer-linked disaccharide glycosides immobilized on the plasmonic optical device surface, label-free detection of oligosaccharide-lectin interactions was carried out.

\section{Materials and Methods}

\subsection{Materials}

To generate the nanoparticle layer, 4,4'-dithiodibutyric acid (DDA), purchased from Aldrich, was used for the formation of a self-assembled monolayer (SAM) on a gold substrate surface. 1-Ethyl-3-(3-dimethylaminopropyl) carbodiimide (EDC), used for the activation of the carboxyl group of DDA, was purchased from Dojindo Laboratories (Kumamoto, Japan). 3-Aminopropyltriethoxysilane ( $\gamma$-APTES), used for the surface modification of silica nanoparticles, was purchased from Shin-Etsu Chemical Co., Ltd., (Tokyo, Japan). Silica nanoparticles (100 nm i.d.), used for preparing the nanoparticle layer, were purchased from Polysciences Inc., (Warrington, PA). The glass substrate (10 $\times 26 \mathrm{~mm}^{2}$; thickness, $1.1 \mathrm{~mm}$ ) was purchased from Fujimoto Science Co., Ltd., (Tokyo, Japan). Ultrapure water $(18.3 \mathrm{M} \Omega-\mathrm{cm})$ procured from Millipore, was used in all the experiments.

For the label-free detection of oligosaccharide-lectin interactions, lectin (wheat germ agglutinin (WGA)) was purchased from Cosmo Bio Co., Ltd., (Tokyo, Japan). Using this lectin, the detection of interactions between chemoenzymatically synthesized oligosaccharides and lectin was evaluated. 5-Aminopentyl- $\beta$-LacNAc, 5-(5'-aminopenta necarboxamido) pentyl- $\beta$-LacNAc, and 2-(2-(2-(2-aminoethoxy)ethoxy)ethoxy)ethyl- $\beta$ LacNAc (LacNAc- $\beta$-TEG-NH ${ }_{2}$ ) were prepared by previously reported methods. ${ }^{(21,22)}$

\subsection{Apparatus}

For the deposition of gold and titanium onto the glass substrate, a sputtering apparatus (L250S-FH) was purchased from Anelva Co., Ltd., (Kanagawa, Japan). The spectrophotometer (USB 4000 UV-VIS; wavelength range, 200-1,100 nm), tungsten halogen light source (LS-1; wavelength range, 360-2,000 nm), and optical fiber probe bundle (R-200-7 UV/VIS; fiber core diameter, $200 \mu \mathrm{m}$; wavelength range, 250-800 nm) used to evaluate the optical characteristics of the LSPR-based plasmonic optical device were purchased from Ocean Optics (Dunedin, USA).

\subsection{Analytical methods}

The ESI-MS spectra were measured using a JMS-T100LC mass spectrometer. ${ }^{1} \mathrm{H}$ NMR spectra were recorded on a JEOL lambda-500 spectrometer at $500 \mathrm{MHz}$, while ${ }^{13} \mathrm{C}$ NMR spectra were recorded using the same instrument at $125 \mathrm{MHz}$. Chemical shifts are expressed in $\delta$ relative to sodium 3-(trimethylsilyl) propionate as an external standard. 


\subsection{Synthesis of divalent LacNAc glycoside carrying disulfide group}

In this study, the divalent LacNAc glycosides with different spacers were synthesized using the chemoenzymatic synthesis method ${ }^{(21,22)}$ as follows (Fig. 1.).

2.4.1 Synthesis of bis-5-(3'-thiobutanecarboxamide)pentyl- $\beta$-LacNAc [(LN-AP$\left.T B A)_{2}\right]$

4,4'-Dithiodibutyric acid (20 $\mathrm{mg}, 0.085 \mathrm{mmol})$, HBTU (65 $\mathrm{mg}, 0.17 \mathrm{mmol}$ ) and DIEA $(150 \mu \mathrm{L}, 0.86 \mathrm{mmol})$ were dissolved in DMSO $(1.9 \mathrm{~mL})$. 5-Aminopentyl- $\beta$ LacNAc $(80 \mathrm{mg}, 0.17 \mathrm{mmol})$ was added to the solution with continuous stirring at room temperature for $1 \mathrm{~h}$. The reaction mixture was loaded onto a Bio-Gel P-2 column (5.0 $\mathrm{cm} \times 90 \mathrm{~cm}$ ). The column was developed with $\mathrm{H}_{2} \mathrm{O}$ at a flow rate of $1.5 \mathrm{~mL} / \mathrm{min}$ and a fraction size of $15 \mathrm{~mL} /$ tube. An aliquot from fractions 51-62 was then concentrated and dissolved in $1.0 \mathrm{~mL}$ of $10 \% \mathrm{MeOH}$ and then loaded onto a Sep-Pak ${ }^{\circledR} \mathrm{C}-18$ column $(2.0 \mathrm{~cm} \times 5.0 \mathrm{~cm})$ developed with the same solvent. The column was washed with $40 \% \mathrm{MeOH}(75 \mathrm{~mL})$, and then eluted with $50 \% \mathrm{MeOH}(75 \mathrm{~mL})$. The eluate was concentrated and lyophilized; (LN-AP-TBA) $)_{2}$ was obtained in a total yield of $55 \%$ (53 mg) based on 4,4'-dithiodibutyric acid. HRESIMS: $\mathrm{m} / z$ 1161.46590 [M $+\mathrm{Na}]^{+}$(calcd for $\left.\mathrm{C}_{46} \mathrm{H}_{82} \mathrm{~N}_{4} \mathrm{Na}_{1} \mathrm{O}_{24} \mathrm{~S}_{2}, 1161.46581\right)$; ${ }^{1} \mathrm{H}$ NMR $\left(\mathrm{D}_{2} \mathrm{O}, 500 \mathrm{MHz}\right): \delta 4.55\left(\mathrm{~d}, 2 \mathrm{H}, \mathrm{J}_{1,2} 7.5\right.$ $\mathrm{Hz}, \mathrm{H}-1), 4.50$ (d, 2H, J $\left.1^{\prime}, 2^{\prime} 8.0 \mathrm{~Hz}, \mathrm{H}-1^{\prime}\right), 4.01$ (2H, H-6b), 3.95 (2H, H-4'), $3.91(2 \mathrm{H}$, H-a $), 3.86$ (2H, H-6a), 3.79-3.72 (12H, H-6'ab, H-3, H-5', H-4, H-2), 3.69 (2H, H-3'), 3.64-3.55 (6H, H-a, H-5, H-2'), 3.20 (4H, H-e), 2.77 (4H, H- $\alpha), 2.38$ (4H, H- $\gamma$ ), 2.06 (s, $\left.6 \mathrm{H}, \mathrm{CH}_{3} \mathrm{CONH}-\right), 2.01$ (4H, H- $\beta$ ), 1.59 (4H, H-b), 1.54 (4H, H-d), $1.36(4 \mathrm{H}, \mathrm{H}-\mathrm{c}) ;{ }^{13} \mathrm{C}$

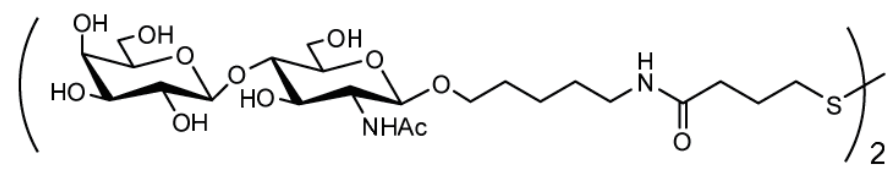

(a)

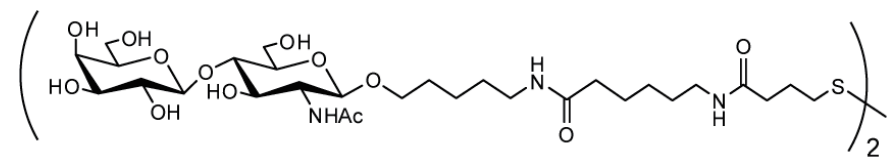

(b)

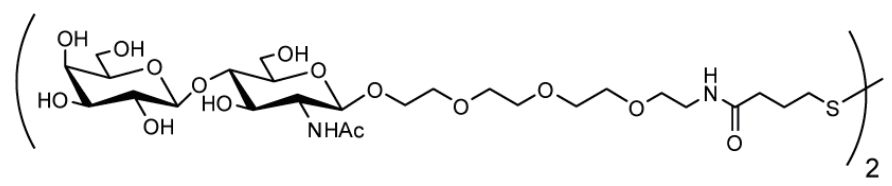

(c)

Fig. 1. Chemical structures of divalent LacNAc glycosides. (a) (LN-AP-TBA) $)_{2}$, (b) (LN-AP-AHTBA $)_{2}$, and (c) (LN-TEG-TBA) . $^{2}$ 
NMR ( $\left.\mathrm{D}_{2} \mathrm{O}, 125 \mathrm{MHz}\right): \delta 178.5$ (-CONH-), $177.1\left(\mathrm{CH}_{3} \mathrm{CONH}-\right), 105.7$ (C-1'), 103.9 (C-1), 81.4 (C-4), 78.2 (C-5'), 77.6 (C-5), 75.4 (C-3), 75.3 (C-3'), 73.8 (C-2'), 73.1 (C-a), 71.4

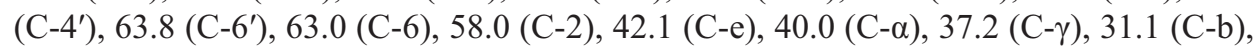
30.8 (C-d), 27.6 (C- $\beta), 25.4$ (C-c), $25.1\left(\mathrm{CH}_{3} \mathrm{CONH}-\right)$.

2.4.2 Synthesis of bis-5-[5'-(3"-thiobutanecarboxamido)pentanecarboxamido] pentyl- $\beta$-LacNAc $\left[(L N-A P-A H-T B A)_{2}\right]$

(LN-AP-AH-TBA) 2 was synthesized by the addition of 5-(5' -aminopentanecarboxamido)pentyl- $\beta$-LacNAc to $4,4^{\prime}$-dithiodibutyric acid in a similar manner. (LN-AP-AH-TBA) $)_{2}$ was obtained in a total yield of $69 \%(57 \mathrm{mg})$. HRESIMS: $m / z$ 1387.63376 [M + Na] ${ }^{+}$(calcd for $\left.\mathrm{C}_{58} \mathrm{H}_{104} \mathrm{~N}_{6} \mathrm{Na}_{1} \mathrm{O}_{26} \mathrm{~S}_{2}, 1387.63393\right)$; ${ }^{1} \mathrm{H}$ NMR $\left(\mathrm{D}_{2} \mathrm{O}\right.$, $500 \mathrm{MHz}): \delta 4.54$ (d, 2H, $\left.\mathrm{J}_{1,2} 7.5 \mathrm{~Hz}, \mathrm{H}-1\right), 4.49$ (d, 2H, J', $\left.7.5 \mathrm{~Hz}, \mathrm{H}-1^{\prime}\right), 3.99(2 \mathrm{H}$, H-6b), 3.94 (2H, H-4'), $3.90\left(2 \mathrm{H}, \mathrm{H}_{-} \mathrm{a}^{\mathrm{b}}\right), 3.84(2 \mathrm{H}, \mathrm{H}-6 \mathrm{a}), 3.19$ (8H, H-e, H-e'), $2.75(4 \mathrm{H}$, $\mathrm{H}-\alpha), 2.36$ (4H, H- $\gamma$ ), 2.25 (4H, H-a'), 2.05 (s, 6H, $\left.\mathrm{CH}_{3} \mathrm{CONH}-\right), 2.00$ (4H, H- $\beta$ ), 1.63-1.49 $\left(16 \mathrm{H}, \mathrm{H}-\mathrm{b}, \mathrm{H}-\mathrm{d}, \mathrm{H}-\mathrm{b}^{\prime}, \mathrm{H}-\mathrm{d}^{\prime}\right), 1.34\left(8 \mathrm{H}, \mathrm{H}-\mathrm{c}, \mathrm{H}-\mathrm{c}^{\prime}\right) ;{ }^{13} \mathrm{C}$ NMR $\left(\mathrm{D}_{2} \mathrm{O}, 125 \mathrm{MHz}\right): \delta 178.4$ and 179.4 (-CONH-), 177.1 ( $\left.\mathrm{CH}_{3} \mathrm{CONH}-\right), 105.7$ (C-1'), 103.8 (C-1), $81.4(\mathrm{C}-4), 78.2\left(\mathrm{C}-5^{\prime}\right)$, 77.6 (C-5), 75.4 (C-3), 75.3 (C-3'), 73.8 (C-2'), 73.1 (C-a), 71.4 (C-4'), 63.8 (C-6'), 63.0 (C-6), 57.9 (C-2), 42.0 (C-e, C-e'), 40.0 (C- $\alpha$ ), 38.5 (C-a'), 37.2 (C- $\gamma), 31.1$ (C-b), 30.8 (C-d, C-d'), 28.4 (C-c'), $27.9\left(\mathrm{C}-\mathrm{b}^{\prime}\right), 27.7$ (C- $\beta$ ), 25.4 (C-c), $25.1\left(\mathrm{CH}_{3} \mathrm{CONH}-\right)$.

\subsubsection{Synthesis of bis-2-\{2-[2-(2-(3'-thiobutanecarboxamido)aminoethoxy)ethoxy]} ethoxy, ethyl- $\beta$-LacNAc [(LN-TEG-TBA $\left.)_{2}\right]$

(LN-TEG-TBA) $)_{2}$ was synthesized by the addition of LacNAc- $\beta$-TEG-NH ${ }_{2}$ to $4,4^{\prime}$ -dithiodibutyric acid in a similar manner. (LN-TEG-TBA) $)_{2}$ was obtained in a total yield of $72 \%$ (37 mg). HRESIMS: $m / z 1341.52898[\mathrm{M}+\mathrm{Na}]^{+}$(calcd for $\mathrm{C}_{52} \mathrm{H}_{94} \mathrm{~N}_{4} \mathrm{Na}_{1} \mathrm{O}_{30} \mathrm{~S}_{2}$, 1341.52920); ${ }^{1} \mathrm{H}$ NMR ( $\left.\mathrm{D}_{2} \mathrm{O}, 500 \mathrm{MHz}\right): \delta 4.60$ (d, 2H, J $\left.\mathrm{J}_{1,2} 8.0 \mathrm{~Hz}, \mathrm{H}-1\right), 4.49$ (d, 2H, J ${ }_{1^{\prime}, 2^{\prime}} 8.0$ Hz, H-1'), 3.41 (4H, H-h), 2.77 (4H, H- $\alpha$ ), 2.40, (4H, H- $\gamma), 2.06$ (6H, s, $\left.\mathrm{CH}_{3} \mathrm{CONH}-\right), 2.02$ (4H, H- $\beta$ ); ${ }^{13} \mathrm{C}$ NMR ( $\left.\mathrm{D}_{2} \mathrm{O}, 125 \mathrm{MHz}\right): \delta 178.8$ (-NHCO-), $177.2\left(\mathrm{CH}_{3} \mathrm{CONH}-\right), 105.7\left(\mathrm{C}-1^{\prime}\right)$, 103.8 (C-1), 81.4 (C-4), 78.2 (C-5'), 77.6 (C-5), 75.4 (C-3), 75.3 (C-3'), 73.8 (C-2'), 72.5 (C-b, C-c, C-d, C-e), 72.3 (C-f), 71.8 (C-a), 71.7 (C-g), 71.4 (C-4'), 63.8 (C-6'), 63.0 (C-6), 57.9 (C-2), 41.8 (C-h), 39.9 (C- $\alpha$ ), 37.1 (C- $\gamma), 27.5$ (C- $\beta), 25.1\left(\mathrm{CH}_{3} \mathrm{CONH}-\right)$.

These divalent LacNAc glycosides carrying a disulfide group are easily immobilized on the plasmonic optical device surface through their disulfide group as mentioned below.

2.5 Fabrication of the LSPR-based plasmonic optical device using a core-shell nanoparticle-layer substrate

The plasmonic optical device was fabricated using a core-shell nanoparticle-layer substrate by a previously reported fabrication procedure. ${ }^{(15-20)}$ From this fabrication procedure, the color generated due to LSPR absorption was monitored in the visible region (Fig. 2). In addition, this plasmonic optical device indicates a specific color due to the LSPR. Furthermore, this color could be observed from the core-shell-structured nanoparticle layer (Fig. 3). 


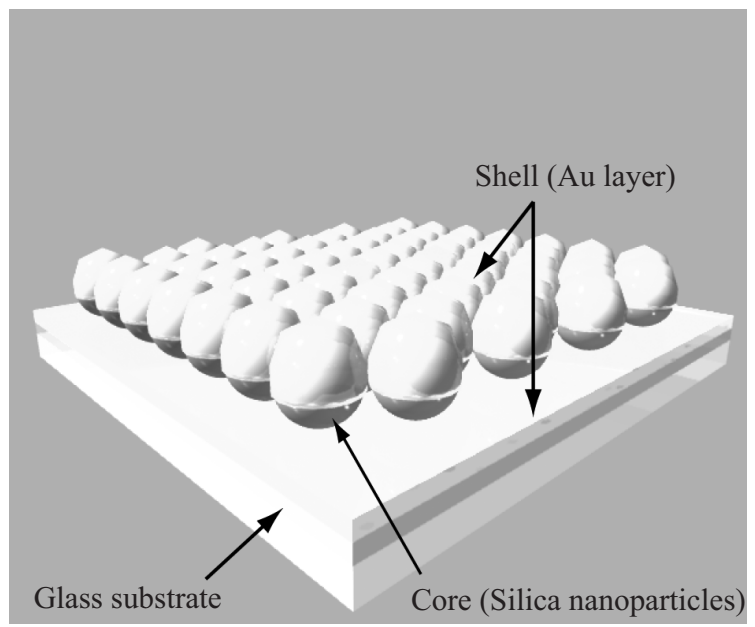

Fig. 2. Construction of the LSPR-based plasmonic optical device.

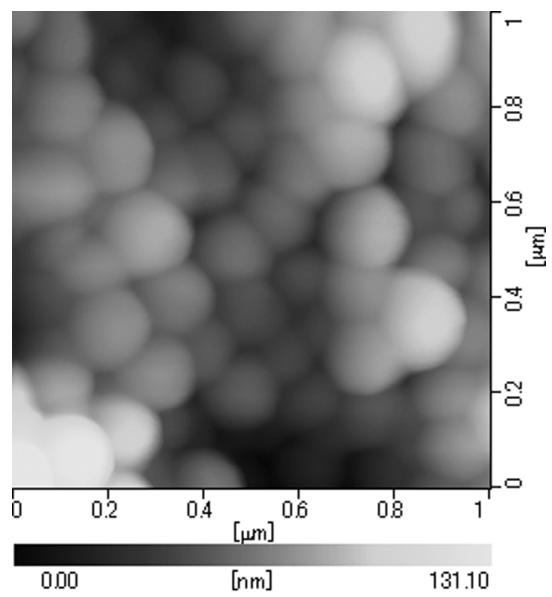

Fig. 3. Surface image of plasmonic optical device obtained by atomic force microscopy (AFM).

\subsection{Experimental setup and detection principles of LSPR-based plasmonic} optical device for detection of oligosaccharide-lectin interactions

The experimental setup of the LSPR-based plasmonic optical device is schematically illustrated in Fig. 4. In this study, a similar optical characterization setup was applied to detect the oligosaccharide-lectin interactions.

The principle by which the LSPR-based plasmonic optical device could detect oligosaccharide-lectin intereactions, which was based on the LSPR-based optical detection principle, is schematically illustrated in Fig. 5. For the detection of lectin, the oligosaccharide was immobilized onto the device surface, and then the sample solution 


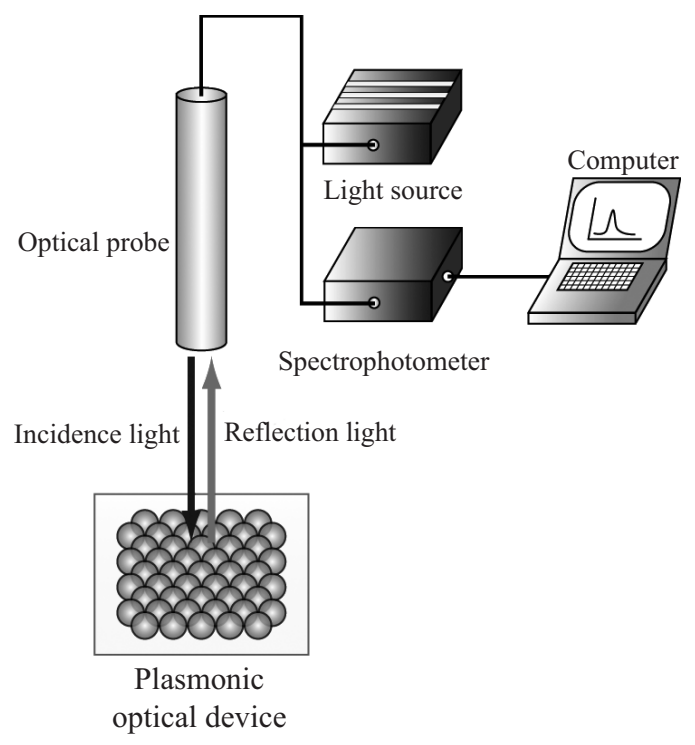

Fig. 4. Schematic illustration of experimental setup for LSPR-based plasmonic optical device.

was introduced onto the oligosaccharide-immobilized device surface. Consequently, the LSPR optical characteristics of the LSPR-based optical device were monitored.

In this study, for immobilization of the oligosaccharide, $10 \mu \mathrm{M}$ of the divalent LacNAc glycoside solution, which was diluted with phosphate buffered saline (PBS, $\mathrm{pH}$ 7.4), was introduced onto the device surface for $30 \mathrm{~min}$ at room temperature (RT). After the immobilization, excess divalent glycoside was removed by washing with PBS. Using these immobilization procedures, the label-free detection of oligosaccharide-lectin interaction was carried out.

In addition, for the detection of LacNAc glycoside-WGA interactions, different concentrations of lectin solutions $(1 \mathrm{nM}-10 \mu \mathrm{M})$, which were diluted with PBS (pH 7.4), were introduced onto the spacer-linked LacNAc glycoside immobilized on optical device surfaces for $30 \mathrm{~min}$ at RT. After the interactions, optical characterization was carried out under atmospheric condition.

\section{Results and Discussion}

\subsection{Basic characteristics of the LSPR-based plasmonic optical device}

The LSPR optical characteristics of the plasmonic optical device are shown in Fig. 6. The LSPR absorbance peak was observed at $553 \mathrm{~nm}$. From the LSPR optical absorbance spectrum monitoring, after the LacNAc glycoside-WGA interactions, the absorbance peak wavelength and strength are changed. From these optical changes, specific detection of LacNAc glycoside-WGA interaction was achieved. 


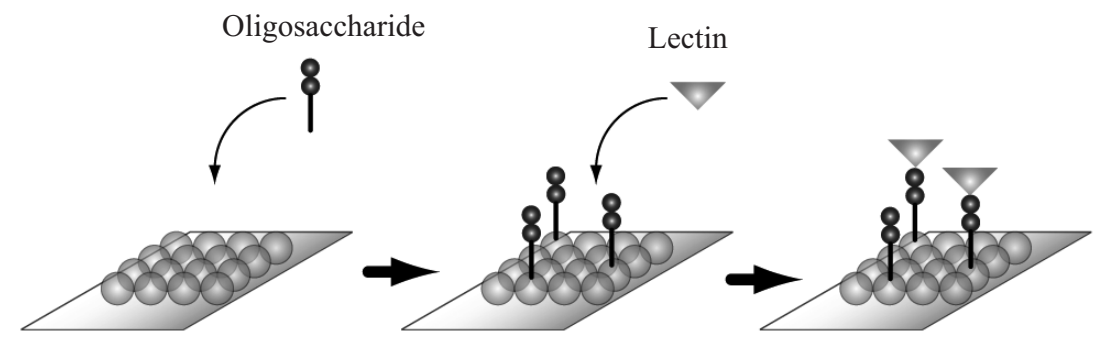

1) Immobilization of

2) Oligosaccharide-lectin interaction

3) Optical characteristics evaluation oligosaccharide

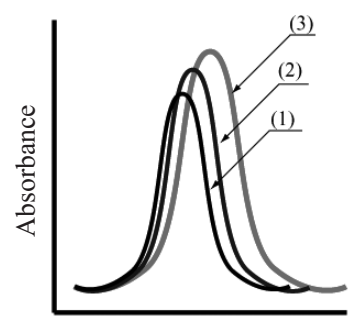

Wavelength

Fig. 5. LSPR-based optical detection principle using plasmonic optical device.

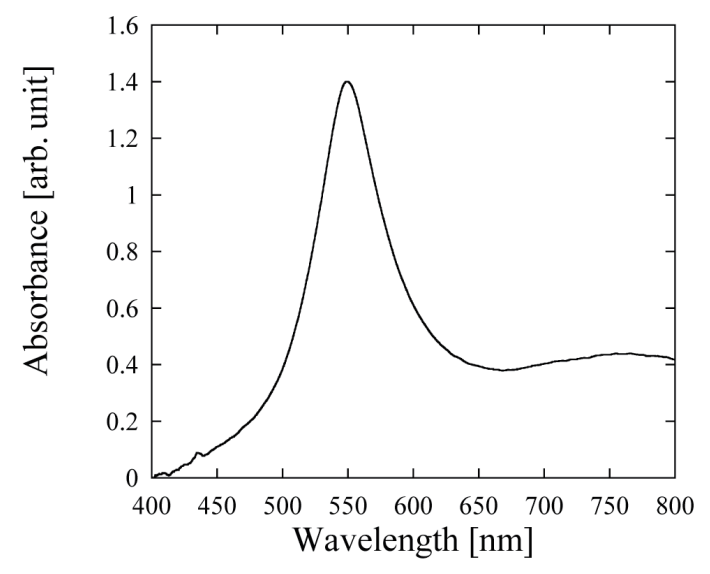

Fig. 6. LSPR optical characteristics of the plasmonic optical device.

\subsection{Label-free detection of oligosaccharide-lectin interaction}

Using the LSPR-based plasmonic optical device, the label-free detection of LacNAc glycoside-WGA interaction was carried out. We observed a change in the LSPR absorbance strength and a shift in the LSPR peak wavelength caused by the oligosaccharide-lectin interaction. These changes in the optical characteristics were 
attributed to the change in the refractive index of the plasmonic optical device surface that was induced by specific LacNAc glycoside-WGA interactions (data not shown). However, a decrease in LSPR optical characteristics (peak wavelength and strength) could be observed. Theoretically, the changes in LSPR optical characteristics with specific interactions were attributed to the increase in peak wavelength and strength caused by the refractive index change. Despite this experiment, decreases in peak wavelength and strength were observed. As a reason for these phenomena, the spacerlinked LacNAc glycoside conformations are affected by LSPR optical characteristics. In this experiment, the spacer-linked LacNAc glycosides carrying a disulfide group were immobilized onto the plasmonic optical device surface directly through their sulfide group. Hence, it became extremely difficult to preserve the stable LacNAc glycoside conformation, while maintaining the immobilization efficiency. Hence, the binding flexibility of LacNAc glycosides was inhibited by their van der Waals attraction. Therefore, further investigations are required to optimize the procedure for immobilizing LacNAc glycosides onto the plasmonic optical device surface and for construction of the optical device.

\subsection{Quantitative determination of lectin using spacer-linked LacNAc glycoside- immobilized LSPR-based plasmonic optical device}

From the above-mentioned experimental results, to improve the immobilization efficiency and conformation flexibility, a divalent LacNAc glycoside and 6-mercapto1-hexanol mixture (mixture ratio, divalent glycoside: 6-mercapto-1-hexanol $=0.5: 9$ ) was prepared for maintaining the oligosaccharide glycoside conformation. Using this mixture, a large portion of 6-mercapto-1-hexanol was immobilized onto the device surface with LacNAc glycosides. Hence, the binding flexibility of oligosaccharides was much improved. In addition, immobilization using this mixture was applied to the above-described immobilization procedure.

The changes in LSPR optical characteristics for WGA using LacNAc glycosides immobilized on the plasmonic optical device is shown in Fig. 7(a). As a result, the changes in LSPR optical characteristics caused by LacNAc glycoside-WGA interaction, which depends on the lectin concentration, could be observed. These LSPR optical characteristics changes were higher than previous experimental results. These results show that the insertion of short-chain thiols (6-mercapto-1-hexanol) on the device surface for LacNAc glycosides immobilization are effectively fulfilling as a background.

From the changes in LSPR optical characteristics for different concentrations of WGA, calibration characteristics were evaluated (Fig. 7(b)). In this study, spacer-linked LacNAc glycosides with different chemical structures were synthesized. The changes in LSPR optical characteristics were different owing to the spacer length of LacNAc glycosides. In this study, from the LSPR optical characteristics, LN-AP-TBA and LNTEG-TBA show high affinity for WGA. In addition, using the LacNAc glycosideimmobilized plasmonic optical device, the detection limit for WGA was $10 \mathrm{nM}$.

However, in this experiment, blue-shift of the LSPR peak wavelength could also be observed. From the previous reports, only the redshift and increment of absorbance strength caused by the antigen-antibody reaction and DNA-DNA hybridization could 
(a)

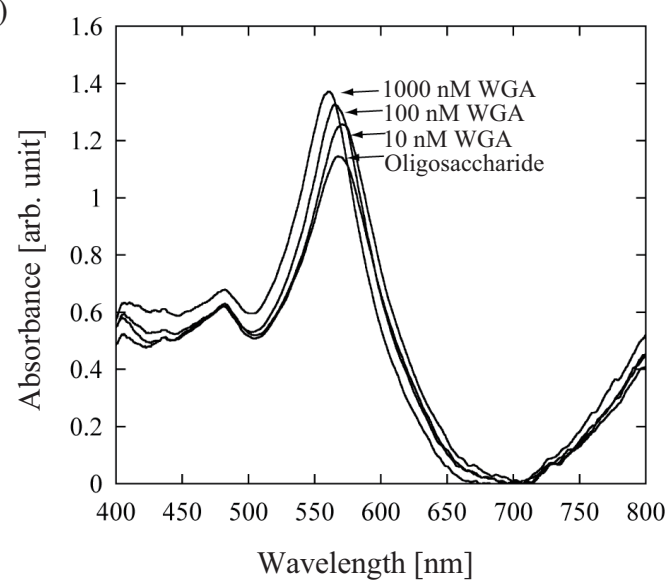

LN-AP-AH-TBA

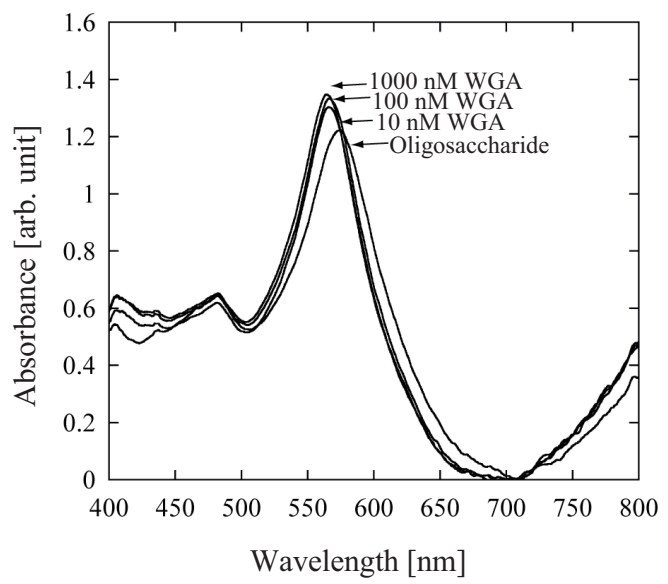

LN-TEG-TBA

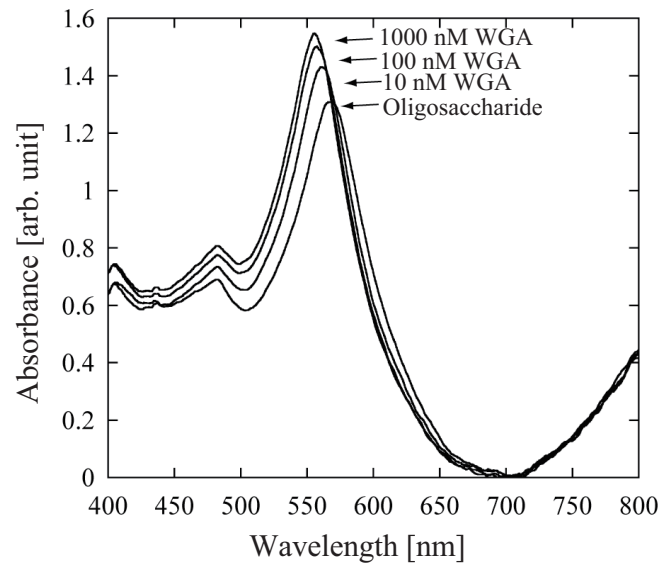

(b)
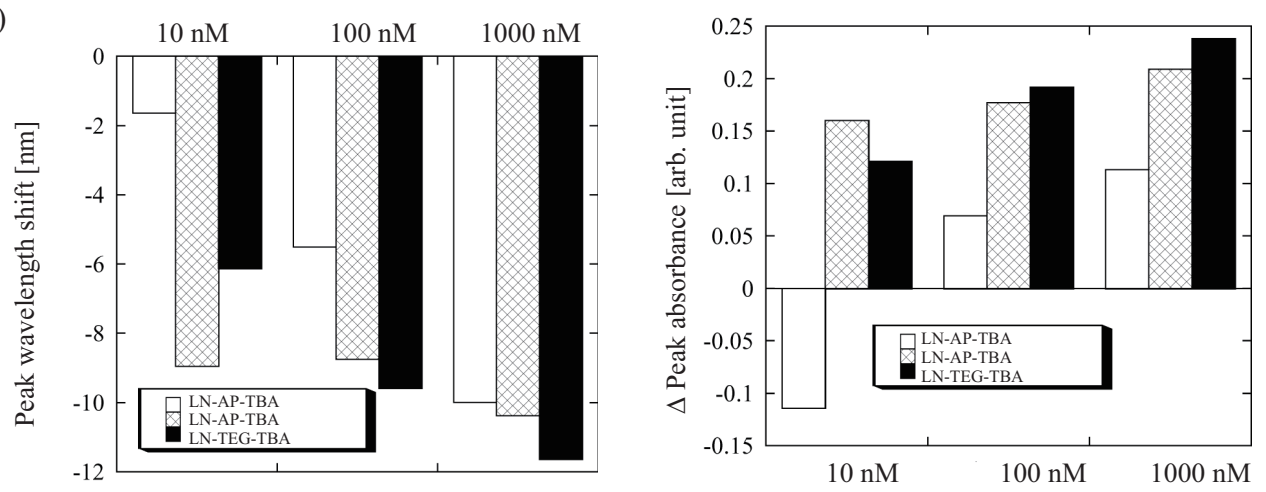

Fig. 7. Changes in LSPR optical characteristics and calibration characteristics for lectin using the oligosaccharide immobilized plasmonic optical device. (a) Changes in LSPR optical characteristics for WGA. (b) Calibration characteristics for WGA. 
be observed. From the viewpoint of the change in LSPR optical characteristics with refractive index, the LSPR peak wavelength shift is attributed to the average refractive index. Theoretically, the LSPR optical characteristics will be affected by the complex refractive index. (Peak shift: real part. Absorbance strength change: imaginary part). Therefore, by the interaction between LacNAc glycoside and WGA, it is considered that the real part of the average refractive index of the LacNAc glycoside-WGA complex decreased. As a result, blue-shift could be observed.

From these results, this plasmonic optical device is sensitive to the average refractive index. Furthermore, the change in LSPR optical characteristics was caused by the average refractive index change. Hence, from a different viewpoint of these results, this plasmonic optical device is applicable to determine the average refractive index of each biomolecular complex.

\section{Conclusions}

We could determine the oligosaccharide-lectin interactions using the plasmonic optical device and chemoenzymatically synthesized divalent LacNAc glycoside-carrying disulfide group. From the previous reports, there are several difficulties in the detection of low-molecular-weight compounds using conventional SPR. However, using this plasmonic optical device has possibilities for the detection of low-molecular-weight compounds. Hence, this result indicates the applicability of this system for future glycomics applications. The LSPR optical characteristics of this device were observed to change owing to the oligosaccharide-lectin interactions. Thus, the plasmonic optical device has great potential for application in the analysis of biofunctions. In addition, for more detailed consideration of the change in LSPR optical characteristics for oligosaccharide-lectin interactions specifically and markedly with high reproducibility, further improvements such as device construction, experimental setup, and immobilization procedure are required.

\section{Acknowledgment}

This study was supported by the Japan Society for the Promotion of Science (JSPS), KAKENHI (Grant-in-Aid for Exploratory Research) (21658116)). In addition, this study was partially supported by the System Development Program for Advanced Measurement and Analysis (SENTAN) of the Japan Science and Technology Agency (JST).

\section{References}

1 M. A. Cooper: Nat. Rev. Drug Discovery 1 (2002) 515.

2 B. L. Wang, Z. M. Li and H. J. Zang: Prog. Chem. 15 (2003) 505.

3 J. A. P. Bons, M. P. van Dieijen-Visser and W. K. W. H. Wodzig: Proteomics Clinical Applications 1 (2007) 1123.

4 H. Andersson and A. van den Berg: Sens. Actuators, B 92 (2003) 315.

5 J. M. Pierce: Proteomics 9 (2009) 5001.

6 W. Vornholt, M. Hartmann and M. Keusgen: Biosens. Bioelectron. 22 (2007) 2983. 
7 M. M. Pedroso, A. M. Watanabe, M. C. Roque-Barreira, P. R. Bueno and R. C. Faria: Microchem. J. 89 (2008) 153.

8 N. Nath and A. Chilkoti: Anal. Chem. 74 (2002) 504.

9 T. S. Ahmadi, S. L. Logunov and M. A. El-Sayed: Size-Dependent Electron Dynamics of Gold Nanoparticles, Nanostructured Materials, eds. V. M. Shalaev and M. Moskovits (American Chemical Society, Washington D. C., 1997) Chap. 10.

10 C. Sönnichsen, B. M. Reinhard, J. Liphardt and A. P. Alivisatos: Nat. Biotechnol. 23 (2005) 741.

11 A. J. Haes and R. P. Van Duyne: J. Am. Chem. Soc. 124 (2002) 10596.

12 D. A. Stuart, C. R. Yonzon, X. Zhang, O. Lyandres, N. C. Shah, M. R. Glucksberg, J. T. Walsh and R. P. Van Duyne: Anal. Chem. 77 (2005) 4013.

13 T. Endo, R. Ikeda, Y. Yanagida and T. Hatsuzawa: Anal. Chim. Acta 611 (2008) 205.

14 Frederix, J. M. Friedt, K. H. Choi, W. Laureyn, A. Campitelli, D. Mondelaers, G. Maes and G. Borghs: Anal. Chem. 75 (2003) 6894.

15 T. Endo, S. Yamamura, N. Nagatani, Y. Morita, Y. Takamura and E. Tamiya: Sci. Technol. Adv. Mater. 6 (2005) 491.

16 T. Endo, K. Kerman, N. Nagatani, Y. Takamura and E. Tamiya: Anal. Chem. 77 (2005) 6976.

17 T. Endo, K. Kerman, N. Nagatani, H. M. Hiep, D. K. Kim, Y. Yonezawa, K. Nakano and E. Tamiya: Anal. Chem. 78 (2006) 6465.

18 T. Endo, K. Kerman, N. Nagatani and E. Tamiya: J. Phys. Condens. Matter 19 (2007) 215201.

19 T. Endo, S. Yamamura, K. Kerman and E. Tamiya: Anal. Chim. Acta 614 (2008) 182.

20 T. Endo, H. Takizawa, Y. Yanagida, T. Hatsuzawa and E. Tamiya: Sens. Mater. 20 (2008) 255.

21 M. Ogata, T. Murata, K. Murakami, T. Suzuki, K. I. P. J. Hidari, Y. Suzuki and T. Usui: Bioorg. Med. Chem. 15 (2007) 1383.

22 M. Ogata, K. I. P. J. Hidari, T. Murata, S. Shimada, W. Kozaki, E. Y. Park, T. Suzuki and T. Usui: Bioconj. Chem. 20 (2009) 538. 